\title{
Valores normativos de força muscular em idosos
}

\author{
Normative values of muscle strength in elderly
}

\author{
Emille Camila de Oliveira Santos ${ }^{1}$ \\ Lucas Lima Galvão ${ }^{1}$ \\ Sheilla Tribess ${ }^{2}$ \\ Joilson Meneguci ${ }^{2}$ \\ Rafaela Gomes Santos ${ }^{1}$ \\ Rízia Rocha Silva ${ }^{1}$ \\ Jair Sindra Virtuoso Junior ${ }^{2}$ \\ Douglas de Assis Teles Santos ${ }^{1}$,
}

\begin{abstract}
Resumo
Objetivo: Identificar os valores normativos da força de membros inferiores e superiores em idosos do Estudo ELSIA. Métodos: A amostra foi composta por 459 sujeitos de ambos os sexos com idade igual ou superior a 60 anos, agrupados por sexo e idade em três grupos, 60-69 anos, 70-79 anos e 80 anos ou mais, moradores da cidade de Alcobaça, Bahia. A força dos membros inferiores foi avaliada através do teste sentar e levantar da cadeira, enquanto a força dos membros superiores foi avaliada através do dinamômetro de preensão manual. Os procedimentos da estatística descritiva foram utilizados para identificar a amostra com a distribuição da frequência, percentil (P10, P20, P30, P40, P50, P60, P70, P80, P90), cálculo de medida de tendência central (média e mediana) e de dispersão (amplitude de variação, desviopadrão) e estatística inferencial (teste Kruskal-Wallis), $\mathrm{p}<0,05$. Resultados: Os resultados do teste de sentar e levantar dos idosos em geral variaram entre 10 e 22,6 repetições na faixa etária de 60-69 anos, 7 e 20 repetições na faixa dos 70-79 anos e 0 e 16 repetições nos $80+$. No teste de preensão manual, seguindo as mesmas faixas etárias, a variação foi de 16,0 e 38,0 kg/f, 14,0 e $36 \mathrm{~kg} / \mathrm{f}$ e 10,0 e 29,0 kg/f. Conclusão: Este estudo possibilitou identificar os valores normativos da força de membros inferiores e superiores dos idosos de Alcobaça, BA. Em ambos os sexos houve uma redução tanto na força dos membros inferiores quanto dos superiores à medida em que a idade foi aumentando, sendo mais significativa a partir dos 80 anos. A partir dos escores obtidos é possível desenvolver parâmetros específicos, para a criação de programas de atividade física que contribua para a manutenção da força dos idosos.
\end{abstract}

Palavras-chave: idoso, saúde, força muscular.

\section{Abstract}

Objective: To identify the normative values of the strength of lower and upper limbs in the elderly of the ELSIA Study. Methods: The sample consisted of 459 subjects of both sexes aged 60 years and older, grouped by sex and age in three groups, 60-69 years, 70-79 years and 80 years or more, residents of the city of Alcobaça, Bahia. The strength of the lower limbs was assessed through the sit and stand test of the chair, while the strength of the upper limbs was assessed by the manual grip dynamometer. Descriptive statistics procedures were used to identify the sample with the frequency distribution, percentile (P10, P20, P30, P40, P50, P60, P70, P80, P90), calculation of central tendency measure (mean and median) and (range of variation, standard deviation) and inferential statistics (Kruskal-Wallis test), $p<0.05$. Results: The results of the sit and stand test of the elderly in general varied between 10 and 22.6 repetitions in the age group of 60-69 years, 7 and 20 repetitions in the range of $70-79$ years and 0 and 16 repetitions in the 80+. In the manual grip test, following the same age groups, the variation was 16.0 and $38.0 \mathrm{~kg} / \mathrm{f}, 14.0$ and $36 \mathrm{~kg} / \mathrm{f}$ and 10.0 and $29.0 \mathrm{~kg} / \mathrm{f}$. Conclusion: This study made it possible to identify the normative values of the strength of the lower and upper limbs of the elderly in Alcobaça, BA. In both sexes there was a reduction in both lower and upper limb strength as age increased, being more significant at age 80. From the obtained scores it is possible to develop specific parameters for the creation of programs of physical activity that contributes to the maintenance of the strength of the elderly.

Keywords: elderly, health, muscle strength.
Afiliação dos autores

${ }^{1}$ Colegiado de Educação Física, Universidade do Estado da Bahia, Teixeira de Freitas, Bahia, Brasil. Teixeira de Freitas, Bahia, Brasil.
Universidade Federal do Triângulo Mineiro, Uberaba, Minas Gerais, Brasil.

${ }^{*}$ Autor correspondente

Colegiado de Educação Física Universidade do Estado da Bahia, Av. Kaikan, s/n, Setor universitário, CEP: 45992-255, Teixeira de Freitas, Bahia, Brasil.

e-mail: datsantos@uneb.br

Conflito de interesses

Os autores declararam não haver conflito de interesses.

Processo de arbitragem

Recebido: 13/10/2018 Aprovado: 27/12/2018 


\section{Introdução}

O envelhecimento populacional vem ocorrendo em um ritmo mais rápido nos últimos anos, e esse fenômeno tem ocorrido em todo o mundo, e ainda é previsto entre 2015 e 2050 um aumento na população com mais de 65 anos na Europa de $23 \%$ para $28 \%$, América do Norte de $18 \%$ para $23 \%$ e até o mesmo ano a Ásia, América Latina, Caribe e Oceania terão mais de $18 \%$ de sua população com a mesma idade ${ }^{1}$.

No Brasil, a população idosa é o segmento populacional que mais cresce, estima-se $4 \%$ ao ano entre 2012 a 2022, e em 2000 a população com 60 anos ou mais de idade era de 14,2 milhões, em 2010 passou para 19,6 milhões, a estimativa para 2030 é de 41,5 milhões e 73,5 milhões em $2060^{2}$. O aumento rápido da população idosa traz impactos para a sociedade como alterações na vida dos indivíduos, na estrutura familiar, nas demandas por políticas públicas e na distribuição de recursos na sociedade.

Em decorrência do aumento da idade, o organismo sofre com o declínio de suas funcionalidades como as capacidades funcionais e metabólicas, dentre estas estão as alterações no sistema musculoesquelético, que são apontadas como uma das que mais influenciam, de forma significativa, na qualidade de vida dos idosos.

A medida em que o corpo envelhece, a gordura corporal tende a aumentar e a massa muscular magra diminui, essa perda relacionada à idade, denomina-se "sarcopenia" processo envolvendo diferentes fatores, 0 que inclui as alterações hormonais, unidade motora remodelada, inatividade física, nutrição inadequada e diminuição da síntese proteica ${ }^{5}$.

A perda de força muscular relacionada a idade, está sendo conceituada como dinapenia ${ }^{5}$. Estudos demonstram que além da perda da massa muscular, outros fatores estão associados à perda da força, como déficits na ativação neural e reduções na capacidade intrínseca de geração de força muscular, ${ }^{6,7}$.

A perda da massa muscular é mais evidente nos membros inferiores e a força muscular é imprescindível para manter as capacidades funcionais, e à medida que o indivíduo envelhece, torna-se ainda mais importante, pois a perda da força é um fator limitante para a execução de tarefas básicas da vida diária, podendo levar o idoso à incapacidade funcional ${ }^{8}$. O teste de sentar e levantar da cadeira, componente do Senior Fitness Test (STF), desenvolvido por Rikli e Jones ${ }^{9}$, tem sido comumente utilizado para avaliar a foça de membros inferiores. Este é descrito como indicador de incapacidade funcional ${ }^{08}$.

A força de preensão manual, além de predizer a força de membros superiores, também tem sido utilizada como indicadora de força global $^{10}$. Essas medidas têm sido investigadas, pois demostram ser um importante preditor de diversos fatores relacionados à saúde na população idosa, incluindo estado nutricional e fragilidade ${ }^{11}$.

Praticar exercícios regularmente ajuda na diminuição dos efeitos deletérios do envelhecimento e colabora para a manutenção da capacidade física do idoso, porém, o desafio é pensar além desses benefícios trazidos às pessoas idosas, como a adesão à prática ${ }^{13}$. Nesse caso, há a necessidade de intervenções rápidas e adequadas do Estado, através de implementação e implantação de políticas públicas fundamentais ${ }^{12}$

Para a implantação de programas de atividade física, devese levar em consideração a avaliação da aptidão física do idoso, possibilitando a detecção de possíveis déficits funcionais, sendo os valores normativos, uma ferramenta essencial para auxiliar na prescrição de exercício físico ${ }^{13}$.

No Brasil foram encontrados estudos sobre valores normativos de força para idosos, específicos apenas nas regiões sul e sudeste ${ }^{14,15}$, no entanto, até o presente momento, não encontramos estudos desenvolvidos na região nordeste. Assim, o objetivo do presente estudo foi identificar os valores normativos da força de membros inferiores e superiores em idosos do Estudo ELSIA.

\section{Métodos}

Estudo observacional, do tipo analítico com delineamento transversal utilizando métodos exploratórios surveys eteste de desempenho físico, realizado no município de Alcobaça, localizada na região do Extremo Sul, do Estado da Bahia.
O presente estudo faz parte do Estudo Longitudinal de Saúde do Idoso de Alcobaça - BA (ELSIA), projeto de pesquisa foi desenvolvido em conjunto entre a Universidade do Estado da Bahia (UNEB) e a Universidade Federal do Triângulo Mineiro (UFTM).

Foram encontrados 743 idosos cadastrados na Estratégia de Saúde da Família, destes 68 idosos se recusaram a participar da pesquisa, 58 foram excluídos por não atenderem os critérios de inclusão e 158 idosos não foram localizados depois de três tentativas.

A amostra foi composta por 459 pessoas de ambos os sexos com idade igual ou superior a 60 anos para o teste de preensão manual, e de 456 para o teste de sentar e levantar. As pessoas selecionadas não deviam conter comprometimento cognitivo grave no Mini Exame do Estado Mental (MEEM), desenvolvido originalmente por Folstein et al. ${ }^{16}$ e adaptado para população brasileira por Almeida ${ }^{17}$; não possuir dificuldade grave na acuidade visual e auditiva, não fazer uso de cadeiras de rodas, não possuir sequelas graves de acidente vascular encefálico (AVE) com perda localizada de força e não possuir doença em estágio terminal.

Para a visita domiciliar os pesquisadores utilizaram como referência dados disponibilizados pela Secretaria Municipal de Saúde de Alcobaça. O contato foi realizado com os idosos por meio de visita domiciliar, informando dos objetivos e solicitando a participação deles na pesquisa, ocorrendo de forma voluntária. Depois de aceito foi entregue um termo de consentimento livre e esclarecido (TCLE) e submetidos a um questionário, aplicado em forma de entrevista, por acadêmicos e profissionais de Educação Física, devidamente treinados. A coleta de dados teve duração de julho a setembro de 2015.

O roteiro de entrevista foi previamente testado em estudo piloto (para identificação dos índices psicométricos), sendo construído mediante a composição de outros instrumentos abordando características sociodemográficas: renda; estado civil (solteiro/divorciado/separado/casado/viúvo); ocupação (aposentado e trabalha/aposentado/do lar/pensionista/trabalho remunerado), indicadores de saúde: enfermidades; sintomatologia depressiva ${ }^{18}$; atividade física ${ }^{19}$; transtornos cognitivos $^{16}$; incapacidade funcional nas atividades da vida diária $^{20}$ (AVD) e instrumentais ${ }^{21}$ (AIVD); risco de desnutrição ${ }^{22}$; percepção de saúde (positiva/negativa); quedas (não/sim); hospitalização (não/sim) e comportamento sedentário ${ }^{23}$.

A força dos membros inferiores foi avaliada através do teste sentar e levantar realizado em cadeira, com o maior número de repetições possíveis durante 30 segundos, este teste faz parte da bateria SFT ${ }^{09}$. Para avaliar a força de preensão manual foi utilizado um dinamômetro SAEHAN Corporation SH5001, Korea, o aparelho foi ajustado de acordo com o tamanho da mão do idoso. O teste foi realizado utilizando o braço que o indivíduo considerou com mais força ${ }^{24}$. Durante a execução do teste, o indivíduo permaneceu em pé, com o braço afastado do corpo e o cotovelo em extensão. Duas tentativas foram realizadas com uma pausa de um minuto. O maior valor obtido foi considerado para análise ${ }^{24}$. A medida da força foi estabelecida em quilogramas/força $[\mathrm{kg} / \mathrm{f}]$.

Para confecção do banco de dados foi utilizado o software Epidata, versão $3.1 \mathrm{~b}$, e as análises por meio do software estatístico SPSS 20.0 (Statistical Package for the Social Sciences). A normalidade dos dados foi verificada pelo teste Kolmogorov-Smirnov. O teste Kruskal-Wallis e ANOVA oneway foi utilizado para comparar as características dos idosos, seguido dos testes post-hoc deTukey ou Dunn respectivamente entre os grupos das faixas etárias. Foi adotado o nível de significância de $p<0,05$. Foi utilizada estatística descritiva dos percentis $\mathrm{P} 10, \mathrm{P} 20, \mathrm{P} 30, \mathrm{P} 40, \mathrm{P} 50, \mathrm{P} 60, \mathrm{P} 70, \mathrm{P} 80, \mathrm{P} 90$ para ambos os sexos e idades.

Este estudo foi aprovado pelo Comitê de Ética em Pesquisa da UFTM sob o parecer número 966.983 e respeitou os procedimentos éticos ditados pela Declaração de Helsinki.

\section{Resultados}

A amostra deste estudo foi composta por 459 idosos, destes, 287 eram do sexo feminino e 172 do sexo masculino, com faixa etária entre 60 e 97 anos, e média de idade de 70,15 $\pm 8,22$ anos. A média etária para as mulheres era de $69,76 \pm$ 8,05 anos e $70,80 \pm 8,48$ anos para os homens. Dos 459,456 
fizeram o teste de sentar e levantar. A Tabela 1 apresenta as características dos idosos.

Tabela1

Características dos participantes.

\begin{tabular}{|c|c|c|c|c|c|}
\hline & $\begin{array}{c}\text { Total } \\
(\mathrm{n}=459)\end{array}$ & $\begin{array}{c}60-69 \\
(n=254) \\
\end{array}$ & $\begin{array}{c}70-79 \\
(n=136)\end{array}$ & $\begin{array}{c}\geq 80 \\
(n=69)\end{array}$ & $\mathrm{p}^{\#}$ \\
\hline $\begin{array}{l}\text { Massa } \\
\text { corporal }(\mathrm{kg})\end{array}$ & $\begin{array}{c}67,86 \pm \\
14,13\end{array}$ & $\begin{array}{c}69,71^{\mathrm{a}} \pm \\
14,44\end{array}$ & $\begin{array}{c}68,16^{\mathrm{d}} \pm \\
13,13\end{array}$ & $\begin{array}{l}60,61^{\mathrm{a}, \mathrm{d}} \\
\pm 12,66\end{array}$ & \\
\hline Estatura $(\mathrm{cm})$ & $\begin{array}{c}157,73 \pm \\
9,63\end{array}$ & $\begin{array}{c}158,13^{\mathrm{b}} \\
\pm 9,20\end{array}$ & $\begin{array}{c}158,75^{\mathrm{e}} \pm \\
9,92\end{array}$ & $\begin{array}{c}154,29^{\mathrm{b}, \mathrm{e}} \\
\pm 9,96\end{array}$ & \\
\hline IMC $\left(\mathrm{kg} / \mathrm{m}^{2}\right)$ & $\begin{array}{c}27,30 \pm \\
5,39\end{array}$ & $\begin{array}{c}27,90^{\circ} \pm \\
5,58\end{array}$ & $\begin{array}{c}27,10 \pm \\
5,04\end{array}$ & $\begin{array}{c}25,49^{\circ} \pm \\
5,00\end{array}$ & \\
\hline $\begin{array}{l}\text { Comportamento } \\
\text { Sedentário total } \\
\text { (min) }\end{array}$ & $\begin{array}{c}433,67 \pm \\
162,44\end{array}$ & $\begin{array}{c}413,22^{\dagger} \\
\pm \\
158,40\end{array}$ & $\begin{array}{c}443,95 \pm \\
155,37\end{array}$ & $\begin{array}{c}487,85^{\dagger} \\
\pm 177,63\end{array}$ & \\
\hline $\begin{array}{l}\text { Nível de Atividade } \\
\text { Física total (min) }\end{array}$ & $\begin{array}{c}364,82 \pm \\
574,54\end{array}$ & $\begin{array}{c}432,13^{g} \\
\pm \\
\pm 03,93\end{array}$ & $\begin{array}{l}361,90^{g, i} \pm \\
610,15\end{array}$ & $\begin{array}{l}126,51^{\mathrm{h}, \mathrm{i}} \\
\pm 236,03\end{array}$ & \\
\hline Renda em valor & $\begin{array}{l}2188,11 \pm \\
3173,45\end{array}$ & $\begin{array}{c}2110,28 \\
\pm \\
3756,49\end{array}$ & $\begin{array}{c}2332,371 \\
4 \pm \\
2158,39\end{array}$ & $\begin{array}{c}2189,75 \\
\pm \\
2467,96\end{array}$ & \\
\hline \multicolumn{6}{|l|}{ Sofreu queda } \\
\hline Não & $\begin{array}{c}336 \\
(71,0 \%) \\
137 \\
(29,0 \%)\end{array}$ & $\begin{array}{c}185 \\
(70,9 \%) \\
76 \\
(29,1 \%)\end{array}$ & $\begin{array}{c}104 \\
(74,3 \%) \\
36 \\
(25,7 \%)\end{array}$ & $\begin{array}{c}47 \\
(65,3 \%) \\
25 \\
(34,7 \%)\end{array}$ & 0,390 \\
\hline \multicolumn{6}{|l|}{ Hospitalização } \\
\hline Não & $\begin{array}{c}393 \\
(83,1 \%) \\
80(16,9 \%)\end{array}$ & $\begin{array}{c}225 \\
(86,2 \%) \\
36 \\
(13,8 \%)\end{array}$ & $\begin{array}{c}110 \\
(78,6 \%) \\
30 \\
(21,4 \%)\end{array}$ & $\begin{array}{c}58 \\
(80,6 \%) \\
14 \\
(19,4 \%)\end{array}$ & 0,124 \\
\hline \multicolumn{6}{|l|}{ Estado civil } \\
\hline $\begin{array}{l}\text { Solteiro } \\
\text { /divorciado } \\
\text { /separado }\end{array}$ & $\begin{array}{c}125 \\
(26,5 \%)\end{array}$ & $\begin{array}{c}75 \\
(28,8 \%)\end{array}$ & $\begin{array}{c}39 \\
(27,9 \%)\end{array}$ & $\begin{array}{c}11 \\
(15,3 \%)\end{array}$ & $<0,001$ \\
\hline Casado & $\begin{array}{c}217 \\
(46,0 \%) \\
130 \\
(27,5 \%)\end{array}$ & $\begin{array}{c}138 \\
(53,1 \%) \\
47 \\
(18,1 \%)\end{array}$ & $\begin{array}{c}61 \\
(43,6 \%) \\
40 \\
(28,6 \%)\end{array}$ & $\begin{array}{c}18 \\
(25,0 \%) \\
43 \\
(59,7 \%)\end{array}$ & \\
\hline $\begin{array}{l}\text { Ocupação } \\
\text { Aposentado e } \\
\text { trabalha }\end{array}$ & 77 (16,3\%) & $\begin{array}{c}45 \\
(17,2 \%)\end{array}$ & $\begin{array}{c}25 \\
(17,9 \%)\end{array}$ & $7(9,7 \%)$ & \\
\hline Aposentado & $\begin{array}{c}311 \\
(65,8 \%)\end{array}$ & $\begin{array}{c}149 \\
(57,1 \%)\end{array}$ & $\begin{array}{c}107 \\
(76,4 \%)\end{array}$ & $\begin{array}{c}55 \\
(76,4 \%)\end{array}$ & $<0,001$ \\
\hline Do lar & $21(4,4 \%)$ & $\begin{array}{c}20 \\
(7,7 \%)\end{array}$ & $1(0,7 \%)$ & $0(0,0 \%)$ & \\
\hline Pensionista & $32(6,8 \%)$ & $\begin{array}{c}18 \\
(6,9 \%)\end{array}$ & $5(3,6 \%)$ & $\begin{array}{c}9 \\
(12,5 \%)\end{array}$ & \\
\hline $\begin{array}{l}\text { Trabalho } \\
\text { remunerado }\end{array}$ & $32(6,8 \%)$ & $\begin{array}{c}29 \\
(11,1 \%)\end{array}$ & $2(1,4 \%)$ & $1(1,4 \%)$ & \\
\hline \multicolumn{6}{|l|}{$\begin{array}{l}\text { Sintomatologia } \\
\text { depressiva }\end{array}$} \\
\hline Ausência & $\begin{array}{c}417 \\
(88,2 \%)\end{array}$ & $\begin{array}{c}236 \\
(90,4 \%)\end{array}$ & $\begin{array}{c}121 \\
(86,4 \%)\end{array}$ & $\begin{array}{c}60 \\
(83,3 \%)\end{array}$ & 0,193 \\
\hline Presença & $56(11,8 \%)$ & $\begin{array}{c}25 \\
(9,6 \%)\end{array}$ & $\begin{array}{c}19 \\
(13,6 \%)\end{array}$ & $\begin{array}{c}12 \\
(16,7 \%)\end{array}$ & \\
\hline \multicolumn{6}{|l|}{ Estado de saúde } \\
\hline $\begin{array}{l}\text { Positiva } \\
\text { Negativa }\end{array}$ & $\begin{array}{c}172 \\
(36,4 \%) \\
300 \\
(63,6 \%)\end{array}$ & $\begin{array}{c}102 \\
(39,1 \%) \\
159 \\
(60,9 \%)\end{array}$ & $\begin{array}{c}48 \\
(34,5 \%) \\
91 \\
(65,5 \%) \\
\end{array}$ & $\begin{array}{c}22 \\
(30,6 \%) \\
50 \\
(69,4 \%)\end{array}$ & 0,353 \\
\hline & & & $p=0,002$ & ${ }^{9} \mathrm{n}<0001$ & \\
\hline
\end{tabular}

$p=0,016$. \#Teste Chi-quadrado.

A Tabela 2 apresenta os resultados do teste de sentar e levantar da cadeira dos idosos e seus percentis de acordo com as faixas etárias. Os valores normativos estão descritos em percentis utilizando os escores de 10 ao 90 , sendo que o primeiro representa o pior e o último o melhor resultado, seguindo uma ordem crescente.

Tabela 2

Valores normativos do teste de sentar e levantar em idosos por idades e sexo.

\begin{tabular}{ccccccccccc}
\hline Faixa & $\mathrm{n}$ & \multicolumn{10}{c}{ Percentis } \\
\cline { 3 - 10 } Etária & & $\mathrm{P} 10$ & $\mathrm{P} 20$ & $\mathrm{P} 30$ & $\mathrm{P} 40$ & $\mathrm{P} 50$ & $\mathrm{P} 60$ & $\mathrm{P} 70$ & $\mathrm{P} 80$ & $\mathrm{P} 90$ \\
\hline Geral & & & & & & & & & & \\
$60-69$ & 253 & 10 & 11 & 13 & 14 & 15 & 16 & 17 & 19 & 22,6 \\
$70-79$ & 135 & 7 & 9 & 11 & 12 & 13 & 14 & 15,2 & 18 & 20 \\
$\geq 80$ & 68 & 0 & 1,6 & 6 & 8 & 9 & 10 & 11,3 & 14 & 16 \\
Mulheres & & & & & & & & & & \\
$60-69$ & 166 & 7,8 & 11 & 13 & 14 & 15 & 16 & 17 & 19 & 21 \\
$70-79$ & 80 & 7,6 & 9 & 11 & 12 & 12,5 & 14 & 15 & 18 & 18,9 \\
$\geq 80$ & 39 & 0 & 0 & 4 & 7 & 8 & 10 & 11 & 13 & 15 \\
Homens & & & & & & & & & & \\
$60-69$ & 87 & 7,8 & 12 & 13 & 15 & 16 & 17 & 19 & 20,4 & 26 \\
$70-79$ & 55 & 7,6 & 10 & 11,8 & 12 & 14 & 14,6 & 17,4 & 19 & 24,4 \\
$\geq 80$ & 29 & 0 & 6 & 8 & 9 & 10 & 11 & 13 & 15 & 18 \\
\hline \multicolumn{7}{l}{ Valores dos percentis expressos em repetições. Faixa etária expressa em anos. }
\end{tabular}

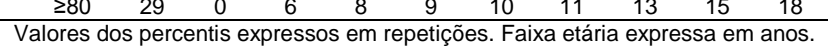

Nos resultados obtidos observa-se um declínio significativo no P10 na faixa etária de $\geq 80$ anos, apresentando nenhuma repetição. O mesmo resultado é observado no P20 do sexo feminino. Além disso, os sexos feminino e masculino apresentaram resultados idênticos no P10 em todas as faixas etárias.

A tabela 3 apresenta os percentis do teste de força de preensão manual, os resultados mostram um declínio da força com o aumento da idade. Os níveis de força do sexo masculino foram superiores aos do sexo feminino. Entretanto, pode-se encontrar resultados idênticos e semelhantes entre faixas etárias 60-69 e 70-79 anos como observado nos P60, P70 e P80 do geral, uma semelhança no P90 do sexo feminino e resultados idênticos no P30 do sexo masculino.

Tabela 3

Valores Normativos do Teste de Preensão Manual em Idosos por idade e sexo.

\begin{tabular}{ccccccccccc}
\hline $\begin{array}{c}\text { Faixa } \\
\text { Etária }\end{array}$ & $\mathrm{n}$ & \multicolumn{10}{c}{ Percentis } \\
\cline { 3 - 11 } & & P10 & P20 & P30 & P40 & P50 & P60 & P70 & P80 & P90 \\
\hline Geral & & & & & & & & & & \\
$60-69$ & 254 & 16,0 & 18,0 & 19,5 & 22,0 & 24,0 & 26,0 & 28,0 & 31,0 & 38,0 \\
$70-79$ & 136 & 14,0 & 15,0 & 17,1 & 19,8 & 22,0 & 26,0 & 28,0 & 31,2 & 36,0 \\
$\quad 80$ & 69 & 10,0 & 13,0 & 14,0 & 16,0 & 18,0 & 20,0 & 24,0 & 26,0 & 29,0 \\
Mulheres & & & & & & & & & & \\
$60-69$ & 167 & 14,0 & 17,0 & 18,0 & 19,0 & 21,0 & 22,0 & 24,0 & 26,0 & 27,0 \\
$70-79$ & 80 & 12,0 & 14,0 & 15,0 & 16,4 & 18,0 & 18,6 & 22,0 & 24,0 & 27,8 \\
$\geq 80$ & 40 & 10,0 & 12,0 & 13,0 & 14,0 & 15,0 & 16,0 & 17,4 & 19,8 & 21,9 \\
Homens & & & & & & & & & & \\
$60-69$ & 87 & 21,8 & 25,2 & 28,0 & 30,2 & 34,0 & 36,0 & 38,0 & 40,0 & 42,0 \\
$70-79$ & 56 & 19,4 & 24,0 & 28,0 & 29,0 & 30,0 & 32,2 & 34,9 & 37,0 & 40,0 \\
$\geq 80$ & 29 & 14,0 & 18,0 & 22,0 & 24,0 & 25,0 & 26,0 & 28,0 & 30,0 & 36,0 \\
\hline \multicolumn{7}{l}{ Valores dos percentis expressos em kg/f. Faixa etária expressa em anos. }
\end{tabular}

\section{Discussão}

O presente estudo permitiu o identificar os valores normativos de membros superiores e inferiores através dos testes de preensão manual e sentar e levantar da cadeira. Foi possível identificar e confirmar que com o avanço da idade há um declínio nos níveis de força, principalmente nos idosos de 80 anos acima.

A força muscular sofre um declínio de $15 \%$ entre os 50 e 70 anos e de $30 \%$ após os 80 anos de idade ${ }^{25}$. Vieira ${ }^{10}$ cita que a perda da força também está associada à diminuição dos níveis hormonais, a qual ocorre com maior prevalência a partir do momento que a mulher entra na menopausa e nos homens a partir da oitava década de vida.

Os valores normativos da força muscular dos membros superiores e inferiores se apresentaram maiores nos homens em relação as mulheres, exceto no P10 do teste de sentar e levantar da cadeira, os quais tiveram resultados iguais. A redução da força de membros inferiores é observada em ambos os sexos à medida em que a idade avança, no entanto parece ser mais acentuada nas mulheres.

Os resultados da força muscular dos membros inferiores são consistentes com os achados dos estudos de Rikli e Jones ${ }^{09}$ as quais apresentam um declínio de $40,8 \%$ no sexo masculino e $44,4 \%$ no sexo feminino da população norte-americana, e Marques et al. ${ }^{26}$ os quais investigaram a população portuguesa apresentando um declínio de $31 \%$ nos homens e $35 \%$ nas mulheres, afirmando que as mulheres apresentam maior risco de dependência prematura do que os homens. Tanto os homens quanto as mulheres do presente estudo apresentaram resultados inferiores no P10 em relação aos estudos citados anteriormente, mas tiveram melhor desempenho no P90 do sexo masculino.

Chung et. al. ${ }^{27}$ mostraram resultados inversos, com uma diminuição de $22,5 \%$ para homens e $14,3 \%$ para as mulheres, isso devido ao fato de as mulheres serem mais ativas do que os homens na população de Hong Kong, e afirmam que é importante desenvolver valores normativos em diferentes regiões devido as diferenças culturais e étnicas.

Os valores dos percentis da força muscular dos membros superiores foram semelhantes nas faixas etárias entre 60-69 e 70-79 anos, tanto no sexo feminino como no sexo masculino. Virtuoso et al. ${ }^{28}$ afirmam que valores abaixo de $30 \mathrm{~kg} / \mathrm{f}$ para homens e $20 \mathrm{~kg} / \mathrm{f}$ para mulheres, indicam prejuízos funcionais e risco de sarcopenia. No presente estudo, pode-se observar valores abaixo do recomendado em todas as faixas etárias. 
Cada vez mais os pesquisadores têm se preocupado em mensurar a força na população idosa, pois evidências tem demonstrado que o declínio da força ocorre de maneira mais acentuada do que o da massa muscular, sendo a força fortemente associada à incapacidade, mortalidade e mais evidente no sexo feminino ${ }^{5,29}$. Os idosos que não são regularmente ativos tendem a diminuir a força de forma acelerada $^{30}$.

Os resultados obtidos mostram-se preocupantes, pois foram encontrados scores abaixo do recomendado, o que apresenta um risco para a saúde. Nessa perspectiva, é importante que a população e o setor público estejam atentos quanto aos riscos, para que medidas de prevenção sejam tomadas.

\section{Conclusão}

Este estudo possibilitou identificar os valores normativos da força de membros inferiores e superiores para idosos de ambos os sexos, com 60 anos acima da cidade de Alcobaça, BA. A partir dos escores obtidos é possível desenvolver parâmetros específicos, para a criação de programas de atividade física que contribua para a manutenção da força dos idosos.

No teste de sentar e levantar da cadeira foram encontrados resultados semelhantes entre homens e mulheres, já no teste de preensão manual, observou-se o mesmo entre as faixas etárias de 60-69 e 70-79 anos do mesmo sexo. Em ambos os sexos houve uma redução tanto na força dos membros inferiores quanto dos superiores à medida em que a idade foi aumentando, sendo mais significativa a partir dos 80 anos. É importante 0 desenvolvimento de valores normativos em diferentes regiões, pois estas apresentam caraterísticas diferentes como cultura e estilo de vida, assim as intervenções podem ser desenvolvidas de acordo com o perfil da região.

\section{Referências}

1. Organização das Nações Unidas. ONU. Cúpula da ONU discute envelhecimento populacional e desenvolvimento sustentável. 2017. Available from: <https://nacoesunidas.org/cupula-da-onu-discuteAvailable from: <https://nacoesunidas.org/cupula-da-onu-discute-
envelhecimento-populacional-e-desenvolvimento-sustentavel/> [2018 out 22].

2. IBGE. Mudança demográfica no Brasil no início do século XXI : subsídios para as projeções da população . Rio de Janeiro: Instituto Brasileiro de Geografia e Estatística; 2015. 156 p.

3. Simões $\mathrm{CC}$ da $\mathrm{S}$. Relações entre as alterações histó ricas na dinâmica demográfica brasileira e os impactos decorrentes do processo de envelhecimento da população. Rio de Janeiro: IBGE; 2016. 113 p.

4. Mazo GZ, Krug R de R, Virtuoso JF, Lopes MA, Tavares AG. Nível de atividade física de idosos longevos participantes de grupos de convivência. Beba. 2012;9(106):4-14.

5. Chang KV, Wu WT, Huang KC, Jan WH, Han DS. Limb muscle quality and quantity in elderly adults with dynapenia but not sarcopenia: An ultrasound imaging study. Exp Gerontol. 2018;108:54-61.

6. Ochala J, Frontera WR, Dorer DJ, Van Hoecke J, Krivickas LS. Single skeletal muscle fiber elastic and contractile characteristics in young and older men. Journals Gerontol - Ser A Biol Sci Med Sci. 2007;62(4):375-81.

7. D'Antona G, Pellegrino MA, Carlizzi CN, Bottinelli R. Deterioration of contractile properties of muscle fibres in elderly subjects is modulated by the level of physical activity. Eur J Appl Physiol. 2007;100(5):603-11.

8. Santos RG, Tribess S, Meneguci J, Da Bastos LLAG, Damião R, Virtuoso JS. Força de membros inferiores como indicador de incapacidade funcional em idosos. Motriz Rev Educ Fis. 2013;19(3 SUPPL):35-42.

9. Rikli RE, Jones CJ. Functional Fitness Normative Scores for Community-
Residing Older Adults, Ages 60-94. J Aging Phys Act. 1999 Apr [cited 2018 Oct 22];7(2):162-81.

10. Vieira M, Souza C, Câmara S, Matos G, Moreira M, Maciel Á. Relação entre força de preensão manual e força de membro inferior em mulheres de meia idade: um estudo transversal. Rev Bras Atividade Física Saúde. 2015;20(5):467

11. Malhotra R, Ang S, Allen JC, Tan NC, Østbye T, Saito Y, et al. Normative Values of Hand Grip Strength for Elderly Singaporeans Aged 60 to 89 Years: A Cross-Sectional Study. J Am Med Dir Assoc. 2016;17(9):864.e1864.e7.

12. Silva MF da, Goulart NBA, Lanferdini FJ, Marcon M, Dias CP. Relação entre os níveis de atividade física e qualidade de vida de idosos sedentários e fisicamente ativos. Rev Bras Geriatr e Gerontol. sedentários e fisican

13. Miranda GMD, Mendes A da CG, Silva ALA da, Miranda GMD, Mendes A da CG, Silva ALA da. Population aging in Brazil: current and future social challenges and consequences. Rev Bras Geriatr e Gerontol. 2016;19(3):507-19.

14. Mazo GZ, Benedetti TRB, Gobbi S, Ferreira L, Lopes MA. Valores normativos e aptidão funcional em homens de 60 a 69 anos de idade. Rev Bras Cineantropometria e Desempenho Hum. 2010;12(5):316-23.

15. Mazo GZ, Petreça DR, Sandreschi PF, Benedetti TRB, Mazo GZ, Petreça $\mathrm{DR}$, et al. Normative values of physical fitness for Brazilian elderly woman aged 60-69 years. Rev Bras Med do Esporte. 2015;21(4):318-22.

16. Folstein MF, Folstein SE, McHugh PR. \&quot;Mini-mental state\&quot;. A practical method for grading the cognitive state of patients for the clinician. J Psychiatr Res. 1975;12(3):189-98.

17. Almeida OP. Mine Exame do Estado Mental e o diagnóstico de demência no Brasil. Arq Neuropsiquiatr. 1998;56(3-B):605-12.

18. Almeida OP, Almeida SA. Confiabilidade da versão brasileira da Escala de Depressão em Geriatria (GDS) versão reduzida. Arq Neuropsiquiatr. 1999 Jun;57(2B):421-6.

19. Benedetti TB, Mazo GZ Barros MVG De. Aplicação do Questionário Internacional de Atividades Físicas para avaliação do nível de atividades físicas de mulheres idosas: validade concorrente e reprodutibilidade testereteste. Rev Bras Ciência e Mov. 2004;12(1):25-34.

20. Lino VTS, Pereira SRM, Camacho LAB, Ribeiro Filho ST, Buksman S. Adaptação transcultural da Escala de Independência em Atividades da Vida Diária (Escala de Katz). Cad Saúde Pública. 2008;24(1):103-12.

21. Santos RL dos, Virtuoso Júnior JS. Confiabilidade da versão brasileira da escala de atividades instrumentais da vida diária. Rev Bras em Promoção da saúde. 2008:21(1):290-6.

22. Guigoz $Y$, Vellas $B$. The Mini Nutritional Assessment (MNA) for grading the nutritional state of elderly patients: presentation of the MNA, history and validation. Nestle Nutr Workshop Ser Clin Perform Programme. 1999;1:3 11; discussion 11-2.

23. Rosenberg DE, Bull FC, Marshall AL, Sallis JF, Bauman AE. Assessment of Sedentary Behavior with the International Physical Activity Questionnaire. J Phys Act Heal. 2008:5(s1):S30-44.

24. DIAS, J. A. et al. Hand grip strength: Evaluation methods and factors influencing this measure. Rev. bras. cineantropom. desempenho hum. 2010; 12(3): 209-216.

25. Mariano ER, Navarro F, Sauaia BA, Oliveira Junior MNS de, Marques RF. Força muscular e qualidade de vida em idosas. Rev Bras Geriatr e Gerontol. 2013;16(4):805-11.

26. Marques EA, Baptista F, Santos R, Vale S, Santos DA, Silva AM, et al. Normative functional fitness standards and trends of portuguese older adults: Cross-cultural comparisons. J Aging Phys Act. 2014;22(1):126-37.

27. Chung PK, Zhao Y, Liu JD, Quach B. Functional fitness norms for community-dwelling older adults in Hong Kong. Arch Gerontol Geriatr. 2016;65:54-62.

28. Virtuoso JF, Balbé GP, Hermes JM, Amorim Júnior EE de, Fortunato AR, Mazo GZ. Grip strength and physical fitness: a predictive study with active elderly. Rev Bras Geriatr e Gerontol. 2014;17(4):775-84.

29. Soares AV, Marcelino E, Maia KC, Borges Junior NG. Relation between functional mobility and dynapenia in institutionalized frail elderly. Einstein. 2017;15(3):278-82.

30. Charlton K, Batterham M, Langford K, Lateo J, Brock E, Walton K, et al. Lean body mass associated with upper body strength in healthy older adults while higher body fat limits lower extremity performance and endurance. Nutrients. 2015;7(9):7126-42. 